\title{
Texture Characterization Using a Curvelet Based Descriptor
}

\author{
Francisco Gómez and Eduardo Romero \\ Bioingenium Research Group - Faculty of Medicine \\ University of Colombia, Bogotá DC - Colombia
}

\begin{abstract}
Feature extraction from images is a key issue in image classification, image representation and content based image retrieval. This paper introduces a new image descriptor, based on the curvelet transform. The proposed descriptor captures edge information from the statistical pattern of the curvelet coefficients in natural images. The image is mapped to the curvelet space and each subband is used for establishing the parameters of a statistical model which captures the subband marginal distributions as well as the dependencies across scales and orientations of the curvelet. Finally, the Kullback-Leibler distance between the statistical parameters is used to measure the distance between images. We demonstrate the effectiveness of the proposed descriptor by classifying a set of texture images, and with a simple nearest neighbour classifier we obtained an accuracy rate of $87 \%$.
\end{abstract}

Keywords: texture characterization, curvelet transform, generalized Gaussian distribution, Kullback-Leibler distance.

\section{Introduction}

The capacity of a mapping to generate features with discriminant characteristics in textures is of paramount importance for the problem of classification and/or retrieval. Typical applications include microscopical or satellite images [1]. Formally, the feature extraction process is thought of as a mapping of an image collection to a characteristic space, which provides a representation where similar images are close and different images are far; this property is known as the discriminating space power. Images projected onto this space are characterized by features which capture some properties of the image, typically some statistical properties from the data. Likewise, a metric for the space is also needed. In the particular case of textures, the most popular characteristic spaces are currently the wavelets, Gabor and DCT transforms [2. Unfortunately, these spaces are sub-optimal for this problem because textures are naturally entailed with geometrical, scale and directional properties which are poorly described with these transforms 3. Some of the features already used for this problem capture information of the energy coefficient distribution and include the total energy, the mean and the variance [2]. However, these features do not reflect correctly the statistical properties of natural images 4. Finally, the usual metrics includes Euclidian or distances between probability density functions such as the

E. Bayro-Corrochano and J.-O. Eklundh (Eds.): CIARP 2009, LNCS 5856, pp. 113 120, 2009.

(C) Springer-Verlag Berlin Heidelberg 2009 
Kullback-Leibler [5]. In these terms the problem of texture characterization consists in constructing a feature with high discriminative power that takes into account the statistical image contents.

The problem of texture characterization with curvelets was already addressed by Semler [6], who studied the performance of several characteristics, namely: the energy, entropy, mean and standard deviation of the curvelet subbands. Results showed significant improvement when comparing with wavelets, but this characterization did not take into account the particular statistical patterns of the curvelet coefficients in texture images [7. Sumana 8] also proposed the curvelet subband mean and variance as features while the Euclidian distance between subbands measured closeness. Results showed again improvement when comparing with Gabor features. However, texture curvelet subbands are not described by simple Gaussians so that mean and variance result insufficient to describe the observed distribution [7].

In this paper we present a new global descriptor, entailed with the previously described properties. The curvelet space is used to capture information about edges which is in fact one of the most discriminating features [9]. These features are the moments of a generalized Gaussian density (GGD) which provides a good approximation to the marginal curvelet subband distribution [7, whilst the Kullback-Leibler distance measures differences between curvelet coefficient distributions. A main contribution of this paper is to demonstrate that taking into account an entire statistical characterization of the curvelet coefficient, results in a highly discriminative, precise and simple descriptor of natural textures. The rest of this paper is organized as follows: Section materials and methods introduces the new feature, Section Results demonstrates the effectiveness of this descriptor in classification tasks. Finally, the last section concludes with a discussion and future work.

\section{Materials and Methods}

The inputs are two images which are curvelet-represented. Frequency subbands are statistically characterized using the moments of a GGD and finally a KullbackLeibler divergence computes the distance between the two representations. This strategy will be further explained hereafter:

\subsection{The Curvelet Transform}

The curvelet transform is a multiscale decomposition 10, developed to naturally represent objects in two dimensions, improving the wavelet limitations in 2D. Curvelets are redundant bases which optimally represent $2 \mathrm{D}$ curves. Besides the usual information about scale and location, already available from a wavelet, each of these frame elements is able to capture information about orientation while also fulfills the parabolic anisotropic scaling law width $\approx$ lengt $h^{2}$, whereby curves at different scale levels conserve their geometrical relationships [10]. A curvelet can be thought of as a radial and angular window in the 


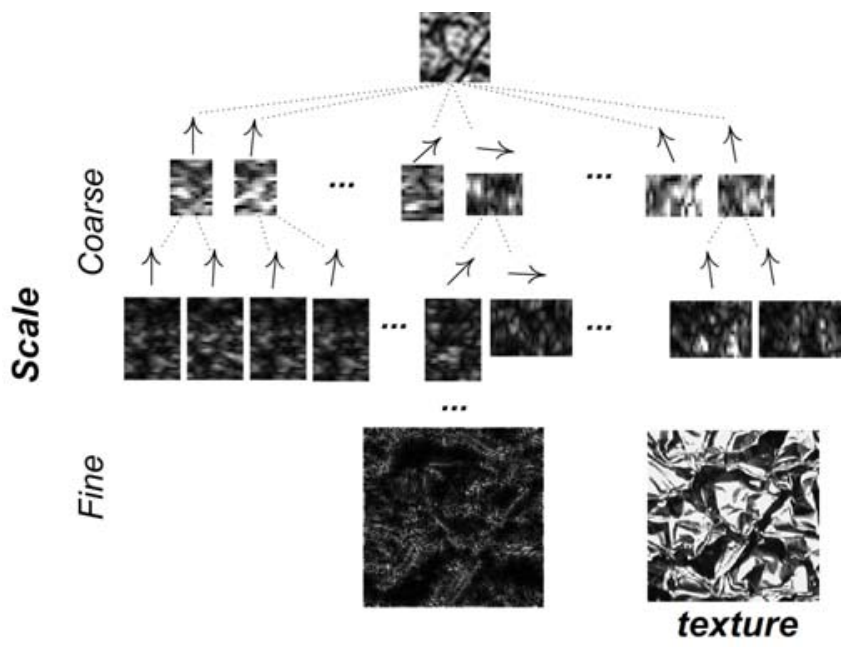

Fig. 1. The figure illustrates a curvelet decomposition of a texture: from top to bottom, increasing levels of detail, from left to right, different orientations

frequency domain, defined in a polar coordinate system. This representation is constructed as the product of two windows: the angular and the radial dyadic frequential coronas. The angular window corresponds to a directional analysis, i.e., a Radon transform, and the radial dyadic window is a bandpass filter whose cut frequencies extract the image information that follows the parabolic anisotropic scaling law [10. Curvelet bases were designed to fully cover the frequency domain, in contrast to other directional multiscale representations such a the Gabor transform 11, with which some information is always lost. Thanks to the anisotropic scale, curvelets adapt much better to scaled curves than Gabor transform, improving the representation at different scales and noise robustness [11. All these statements have been experimentally demonstrated by comparing wavelets, curvelets and Gabor in classification and retrieval tasks 8 .

The curvelet $\varphi_{j, l, k}$ is indexed by scale $j$, orientation $l$ and position $k$, and the curvelet coefficient is simply $c_{j, l, k}=\left\langle f, \varphi_{j, l, k}\right\rangle$, that is to say the projection of the image $f$ over the curvelet basis $\varphi_{j, l, k}$. Typically, the spatial curvelet coefficients with the same scale and orientation are grouped per subbands. The figure [ shows a curvelet multiscale decomposition example.

\subsection{Statistical Characterization}

Psychophysical research has demonstrated that two homogeneous textures are not discriminable if their marginal subband distributions are alike [9]. This fact suggests that these distributions have a highly descriptive capacity, at least for the texture problem. This discriminative power was also experimentally verified for Wavelet and Gabor representations [2]. In the curvelet case, each subband contains information about the degree of occurrence of similar curves within the 


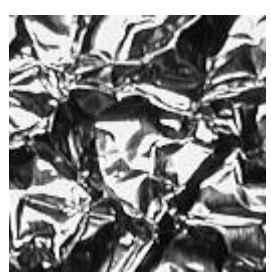

(a) Texture.

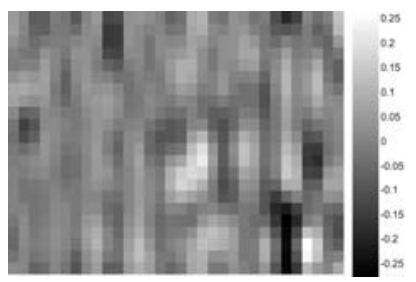

(b) Curvelet Subband

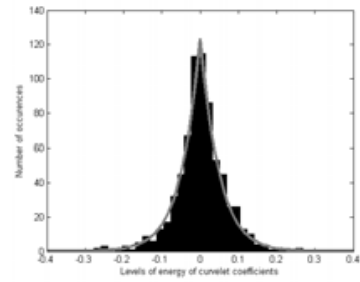

(c) Curvelet histogram.

Fig. 2. Curvelet histogram example (scale 3 and orientation 16)

image, i.e., edge energy levels with similar direction and size. Figure 2 shows a typical example of the curvelet coefficient histogram of an image subband. The kurtosis in this case is about 7.4 so that a Gaussian density is not enough as to match the observed energies. Therefore, the mean and variance calculated from a Gaussian, used in a previous works 6 68 have a very poor descriptive capacity. In general, the curvelet coefficient distribution in natural images is characterized by a sharper peak at zero with smooth tails. This shape is associated to the sparse property of this transformation, i.e., few coefficients have high probability. This leptokurtic pattern has been previously observed in curvelets $7 / 12$ as well as in wavelets $[13$. This work proposes a texture characterization via the marginal distribution of the subband curvelet coefficients, specifically using the parameters of a generalized Gaussian density. Recent experimentation in natural images 7 shows that the generalized Gaussian density provides a good adjustment to the marginal density of the curvelet coefficient within each subband. The GGD reads as $p(x ; \alpha, \beta)=\frac{\beta}{2 \alpha \Gamma(1 / \beta)} e^{-(|x| / \alpha)^{\beta}}$, where $\Gamma(z)=\int_{0}^{\infty} e^{-t} t^{z-1} d t, z>0$ is the Gamma function, $\alpha$ is the variance and $\beta$ is related to the decreasing rate of the GGD. The parameters $\alpha$ and $\beta$ are estimated from the subbband data through Maximum Likelihood, as is detailed in [13. The parameters $(\alpha, \beta)$ may be used as descriptor of the probability density function of the energy levels inside each curvelet subband.

\subsection{Similarity Measure}

The similarity between subband curvelets is measured using the Kullback-Leibler divergence (KLD) of the corresponding GGDs:

$$
D\left(p\left(. ; \alpha_{1}, \beta_{1}\right) \| p\left(. ; \alpha_{2} ; \beta_{2}\right)\right)=\log \left(\frac{\beta_{1} \alpha_{2} \Gamma\left(1 / \beta_{2}\right)}{\beta_{2} \alpha_{1} \Gamma\left(1 / \beta_{1}\right)}\right)+\left(\frac{\alpha_{1}}{\alpha_{2}}\right)^{\beta_{2}} \frac{\Gamma\left(\left(\beta_{2}+1\right) / \beta_{1}\right)}{\Gamma\left(\left(1 / \beta_{1}\right)\right.}-\frac{1}{\beta_{1}}
$$

where $\left(\alpha_{1}, \beta_{1}\right)$ and $\left(\alpha_{2}, \beta_{2}\right)$ are the GGD parameters estimated for each subband. This metric does not require additional normalization and shows good performance in other multiscale domains [13. Finally, under the reasonable assumption that curvelet coefficients in different subbands are independent, the similarity between two images $I_{1}$ and $I_{2}$ is measured as the sum of the distances between corresponding subbands $D\left(I_{1}, I_{2}\right)=\sum_{\forall s} \sum_{\forall \theta} D\left(p\left(. ; \alpha_{1}^{s, \theta} ; \beta_{1}^{s, \theta}\right) \| p\left(. ; \alpha_{2}^{s, \theta} ; \beta_{2}^{s, \theta}\right)\right)$, 
where $\left(\alpha_{1}^{s, \theta}, \beta_{1}^{s, \theta}\right)$ and $\left(\alpha_{2}^{s, \theta}, \beta_{2}^{s, \theta}\right)$, are the GGD parameters estimated for corresponding subbands, i.e., subbands in the same scale $s$ and orientation $\theta$.

\section{$3 \quad$ Experimental Results}

The proposed descriptor was evaluated using the KTH-TIPS1 image texture database. This database provides several variations of scale, pose and illumination and is mainly focused on classification applications; these changes increase the intra-class variability and reduce the inter-class separability, which can increase the difficulty of the classification task compared to typical databases [14]. The data consists of ten texture categories: sandpaper $(s n)$, aluminium foil $(a f)$, styrofoam $(s f)$, sponge $(s p)$, corduroy $(c d)$, linen $(l n)$, cotton $(c t)$, brown bread $(b b)$, orange peel $(o p)$, cracker $(c r)$. These real world images come from different natural scenes and have different orientations and scales. For our experiments, 45 images of each category were converted to gray-scale levels (computed from the luminance component) and cropped to $128 \times 128$. Figure 3 displays examples of the original textures. A real digital curvelet transform with 4 scales and 32 orientations was used, resulting in 66 subbands. The coarsest curvelet level was excluded in order to obtain robustness to changes in illumination. The algorithms are written in Matlab and run on a Intel Xeon X5460 Quad-Core 3.16 $G H z$ with $8 G B$ in RAM.

The objective of the experimentation was to determine the power of description of our feature. Provided that our main goal was to assess the discriminative power of the curvelet descriptor, the feature performance in a multiclass problem was assessed using the most simple classifier, a nearest neighbour, and compared

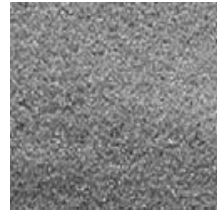

(a) Sandpaper

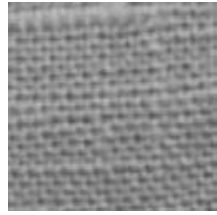

(f) Linen

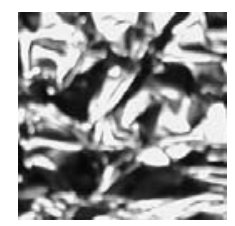

(b) Aluminium

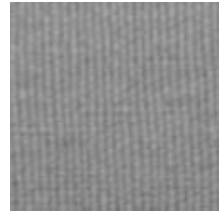

(g) Cotton

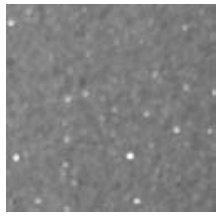

(c) Styrofoam

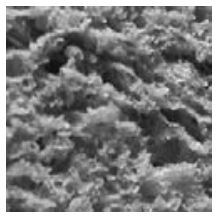

(h)

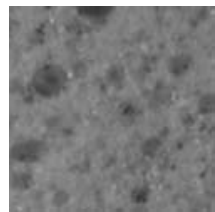

(d) Sponge

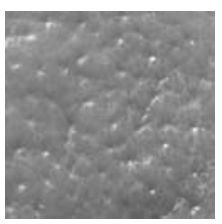

(i) Orange peel

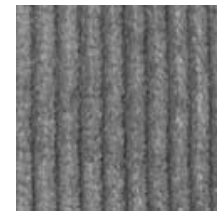

(e) Corduroy

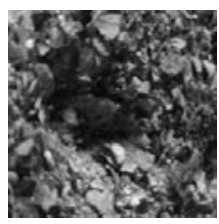

(j) Cracker

Fig. 3. Images example of several textures

${ }^{1}$ http://www.nada.kth.se/cvap/databases/kth-tips/index.html 
Table 1. Confusion matrix for a feature based on energy and Euclidian metric

\begin{tabular}{|c|c|c|c|c|c|c|c|c|c|c|c|c|c|}
\hline & \multicolumn{10}{|c|}{ Assigned } & \multirow{2}{*}{ Total } & \multirow{2}{*}{$\%$ Agree } \\
\hline & & $s n$ & $a f$ & $s f$ & $s p$ & $c d$ & $\ln$ & $c t$ & $b b$ & $o p$ & $c r$ & & \\
\hline \multirow{11}{*}{ True } & $s n$ & 21 & 0 & 14 & \begin{tabular}{|l|}
7 \\
\end{tabular} & 0 & 0 & 0 & 2 & 1 & \begin{tabular}{|l|}
0 \\
\end{tabular} & 45 & 0.47 \\
\hline & $a f$ & 0 & 42 & 1 & 0 & 0 & 0 & 0 & 1 & 1 & 0 & 45 & 0.93 \\
\hline & $s f$ & 5 & 1 & 35 & 3 & 0 & 0 & 0 & 0 & 0 & 1 & 45 & 0.78 \\
\hline & $d p$ & 2 & 0 & 1 & 38 & 2 & 0 & 0 & 1 & 1 & 0 & 45 & 0.84 \\
\hline & $c d$ & 0 & 0 & 0 & 2 & 31 & 0 & 0 & 6 & 6 & 0 & 45 & 0.69 \\
\hline & $\ln$ & 0 & 0 & 0 & 0 & 1 & 42 & 0 & 0 & 1 & 1 & 45 & 0.93 \\
\hline & $c t$ & 0 & 0 & 0 & 1 & 0 & 0 & 40 & 3 & 1 & 0 & 45 & 0.89 \\
\hline & $b b$ & 2 & 0 & 0 & 1 & 1 & 0 & 0 & 36 & 4 & 1 & 45 & 0.80 \\
\hline & $o p$ & 0 & 0 & 1 & 1 & 2 & 0 & 0 & 2 & 27 & 12 & 45 & 0.60 \\
\hline & $c r$ & 0 & 0 & 1 & 0 & 0 & 0 & 0 & 1 & 2 & 41 & 45 & 0.91 \\
\hline & Total & 30 & 43 & 53 & 53 & 37 & 42 & 40 & 52 & 44 & 56 & 450 & 0.78 \\
\hline
\end{tabular}

Table 2. Confusion matrix for a feature based on mean, variance and Euclidian metric

\begin{tabular}{|c|c|c|c|c|c|c|c|c|c|c|c|c|c|}
\hline & \multicolumn{10}{|c|}{ Assigned } & \multirow{2}{*}{ Total } & \multirow{2}{*}{$\%$ Agree } \\
\hline & & $s n$ & $a f$ & $s f$ & $s p$ & $c d$ & $\ln$ & $c t$ & $b b$ & $o p$ & $c r$ & & \\
\hline \multirow{11}{*}{ True } & $s n$ & 29 & 0 & 9 & \begin{tabular}{|l|}
5 \\
\end{tabular} & 0 & 0 & 0 & 2 & \begin{tabular}{|l|}
0 \\
\end{tabular} & \begin{tabular}{|l|}
0 \\
\end{tabular} & 45 & 0.64 \\
\hline & af & 0 & 41 & 1 & 0 & 0 & 0 & 0 & 1 & 0 & 2 & 45 & 0.91 \\
\hline & $s f$ & 4 & 0 & 38 & 2 & 0 & 0 & 0 & 0 & 0 & 1 & 45 & 0.84 \\
\hline & $s p$ & 3 & 0 & 0 & 39 & 2 & 0 & 0 & 0 & 1 & 0 & 45 & 0.87 \\
\hline & $c d$ & 0 & 0 & 0 & 2 & 31 & 0 & 0 & 7 & 5 & 0 & 45 & 0.69 \\
\hline & $\ln$ & 0 & 0 & 0 & 0 & 1 & 44 & 0 & 0 & 0 & 0 & 45 & 0.98 \\
\hline & $c t$ & 1 & 0 & 0 & 0 & 2 & 0 & 41 & 1 & 0 & 0 & 45 & 0.91 \\
\hline & $b b$ & 2 & 0 & 0 & 2 & 1 & 0 & 0 & 35 & 5 & 0 & 45 & 0.78 \\
\hline & $o p$ & 0 & 0 & 0 & 1 & 2 & 0 & 0 & 1 & 36 & 5 & 45 & 0.80 \\
\hline & $c r$ & 0 & 0 & 0 & 0 & 0 & 0 & 0 & 0 & 1 & 44 & 45 & 0.98 \\
\hline & Total & 39 & 41 & 48 & 51 & 39 & 44 & 41 & 47 & 48 & 52 & 450 & 0.84 \\
\hline
\end{tabular}

Table 3. Confusion matrix for our proposed feature: GGD and KLD metric

\begin{tabular}{|c|c|c|c|c|c|c|c|c|c|c|c|c|c|}
\hline & \multicolumn{10}{|c|}{ Assigned } & \multirow{2}{*}{ Total } & \multirow{2}{*}{$\%$ Agree } \\
\hline & & $s n$ & $\overline{a f}$ & $\overline{s f}$ & $s p$ & $c d$ & $\ln$ & $\overline{c t}$ & $\overline{b b}$ & $o p$ & $c r$ & & \\
\hline \multirow{11}{*}{ True } & $s n$ & 31 & 0 & 4 & 5 & 0 & 0 & 0 & 5 & 0 & \begin{tabular}{|l|}
0 \\
\end{tabular} & 45 & 0.69 \\
\hline & $a f$ & 0 & 45 & 0 & 0 & 0 & 0 & 0 & 0 & 0 & 0 & 45 & 1.00 \\
\hline & $s f$ & 3 & 0 & 38 & 1 & 0 & 0 & 0 & 0 & 0 & 3 & 45 & 0.84 \\
\hline & $s p$ & 2 & 0 & 0 & 38 & 2 & 0 & 0 & 3 & 0 & 0 & 45 & 0.84 \\
\hline & $c d$ & 0 & 0 & 0 & 2 & 32 & 0 & 0 & 6 & 3 & 2 & 45 & 0.71 \\
\hline & $\ln$ & 0 & 0 & 0 & 0 & 0 & 44 & 0 & 0 & 1 & 0 & 45 & 0.98 \\
\hline & $c t$ & 0 & 0 & 0 & 0 & 2 & 0 & 43 & 0 & 0 & 0 & 45 & 0.96 \\
\hline & $b b$ & 4 & 0 & 0 & 2 & 0 & 0 & 0 & 39 & 0 & 0 & 45 & 0.87 \\
\hline & $o p$ & 1 & 0 & 1 & 1 & 0 & 0 & 0 & 0 & 42 & 0 & 45 & 0.93 \\
\hline & $c r$ & 0 & 1 & 0 & 0 & 1 & 0 & 0 & 1 & 1 & 41 & 45 & 0.91 \\
\hline & $\overline{\text { Total }}$ & 41 & 46 & 43 & 49 & 37 & 44 & $\overline{43}$ & 54 & 47 & 46 & 450 & 0.87 \\
\hline
\end{tabular}


with other curvelet representation methods, namely: energy of the curvelet subband plus Euclidian metric [68, mean and variance plus Euclidian metric [8] and the herein described proposal GGD plus KLD metric. Sumana 8] has previously compared Gabor, wavelets and curvelets, obtaining a better performance for the latter so that our work is focused on characterizing curvelets. The three classifiers were tested under a Leave-one-out cross-validation, using a single observation from the original sample as the validation data, and the remaining observations as the training data. For the three sets of experiments we computed the corresponding confusion matrix. The confusion matrices for these cases are shown in Tables 1 2] and 3. The correct classification rates of $78 \%, 84 \%$ and $87 \%$ show a high discriminative capacity provided by the curvelet representation, even though we used the simpler classifier. The curvelet descriptor shows a better classification rate in both average and individually for most classes, when compared with mean and variance. Note that textures linen (ln) and cotton (ct) present a high density of lines and are correctly classified in a large number of cases. Likewise, the texture Aluminium (al), which presents gross edges, is correctly classified using the curvelet descriptor. Finally, the confusion matrices show that most misclassifications occur in similar textures, for example, sandpaper (sn) and styrofoam $(s f)$, probably because of the similar edge distributions. In any case, the curvelet descriptor shows less classification errors even in this complicated scenario. These results show that in textures with higher levels of variability, the proposed method outperforms the previous approach. Nevertheless an extensive experimentation is needed to to draw more general conclusions. With respect to the computational complexity, the curvelet implementation runs in $O\left(n^{2} \log (n)\right)$ for $n \times n$ cartesian arrays [10] with a computational time that less than $300 \mathrm{~ms}$ for each image, while the statistical characterization for the curvelet subbands runs in less that 1 second.

\section{Conclusions}

We have introduced a new texture descriptor for images, based on curvelets and a statistical model of the curvelet coefficients in natural images. By applying the curvelet transform and adjusting the levels of energy for each subband to a generalized Gaussian model, we obtain a robust representation which captures the edge distribution at different orientation and scales. Experimental results indicate that the new feature improves classification performance in a multiclass problem when compared with other features, also based on curvelets. Future works includes improving the feature with invariance to rotation and scale and extensive experimentation in large texture databases.

\section{References}

1. Valerie, R.: Introduction to Texture Analysis: Macrotexture, Microtexture and Orientation Mapping. CRC Press, Amsterdam (2000)

2. Randen, T., Hå, J.: Filtering for texture classification: A comparative study. IEEE Trans. Pattern Anal. Mach. Intell. 21(4), 291-310 (1999) 
3. Welland, G.: Beyond Wavelets. Academic Press, San Diego (2003)

4. Wouwer, G.V.D., Scheunders, P., Dyck, D.V.: Statistical texture characterization from discrete wavelet representations. IEEE Transactions on Image Processing 8, 592-598 (1999)

5. Kullback, S.: The kullback-leibler distance. The American Statistician (41), 340$341(1987)$

6. Dettori, L., Semler, L.: A comparison of wavelet, ridgelet, and curvelet-based texture classification algorithms in computed tomography. Comput. Biol. Med. 37(4), 486-498 (2007)

7. Alecu, A., Munteanu, A., Pizurica, A., Cornelis, W.P.J., Schelkens, P.: Informationtheoretic analysis of dependencies between curvelet coefficients, pp. 1617-1620 (2006)

8. Sumana, I., Islam, M., Zhang, D., Lu, G.: Content based image retrieval using curvelet transform. In: Proc. of IEEE International Workshop on Multimedia Signal Processing MMSP, pp. 11-16 (2008)

9. Field, D.J.: Scale-invariance and self-similar 'wavelet' transforms: an analysis of natural scenes and mammalian visual systems, pp. 151-193. Elsevier-Health Sciences Division (1993)

10. Candes, E., Demanet, L., Donoho, D., Ying, L.: Fast discrete curvelet transforms. Multiscale Modeling and Simulation 5(3), 861-899 (2006)

11. Candes, E.: New multiscale transforms, minimum total variation synthesis: applications to edge-preserving image reconstruction. Signal Processing 82(25), 1519-1543 (2002)

12. Boubchir, L., Fadili, M.J.: Multivariate statistical modeling of images with the curvelet transform. In: Eighth International Conference on Signal Processing and Its Applications - IEEE ISSPA 2005, pp. 747-750 (2005)

13. Do, M., Vetterli, M.: Wavelet-based texture retrieval using generalized gaussian density and kullback-leibler distance. IEEE Transactions on Image Processing 11(2), 146-158 (2002)

14. Kondra, S., Torre, V.: Texture classification using three circular filters. In: ICVGIP, pp. 429-434 (2008) 